\title{
STOCHASTIC RESONANCE AND OPTIMAL CLUSTERING FOR ASSEMBLIES OF ION CHANNELS
}

\author{
G. SCHMID, I. GOYCHUK and P. HÄNGGI \\ Institut für Physik, Universität Augsburg \\ 86135 Augsburg, Germany \\ S. ZENG and P. JUNG \\ Department of Physics and Astronomy and Quantitative Biology Institute, Ohio University \\ Athens, $\mathrm{OH} 45701$
}

Received 19 January 2004

Revised 23 February 2004

Accepted 23 February 2004

\begin{abstract}
We consider the statistical properties of action potentials generated by clusters of sodium and potassium channels due to channel noise and/or external stimulation. Since the fluctuations are related to the cluster size, a size-resonance effect — analog to stochastic resonance - is observed that facilitates optimal decoding of small external stimuli at optimal cluster sizes. Furthermore, in analogy to the coherence resonance effect, the channel-noise induced firing patterns exhibit a resonant-like temporal coherence as a function of the cluster size even in the absence of a periodic stimulus. In the presence of additional synaptic noise, SR occurs only for large cluster sizes which possess suboptimal internal noise levels.
\end{abstract}

Keywords: Excitable membranes; ion channel clusters; channel noise; spiking rate; intrinsic coherence resonance; intrinsic stochastic resonance.

\section{Introduction}

Clustering of ion channels is a frequently observed phenomenon in nature. It occurs naturally in myelinated neurons, where the active sodium channels are concentrated at the nodes of Ranvier acting as signal boosters. But it also occurs in neurons that are not myelinated, e.g. in retinal neurons, where myelination would interfere with vision [1]. The mechanisms for clustering of ion channels and receptors are subject of current investigations in neurobiology and cell-biology. Some theories are based on attractive interactions between the channel proteins [2], others on the formation of micro-domains ("rafts") in the membrane with higher affinity for signaling proteins, and still others the involvement of the cytoskeleton anchoring the channels by a sub-membrane undercoat [1]. In this paper we review recent papers that explore the novel idea that ion channel clustering enhances neuronal 
information encoding $[3,4]$. In these papers, it has been shown that small signals may be better detected by smaller clusters of ion channels. The essential idea is based on the theory of stochastic resonance $[5,6]$. A subthreshold signal can be encoded by an excitable system when an optimal amount of noise is added to the signal. In the case of ion channel clusters, the fluctuations are generated by the thermal opening and closing of ion channels. The number of ion channels determines the amplitude of the fluctuations of the membrane potential. Thus instead of tuning the noise, one can tune the size, resulting in a system-size resonance first reported in $[3,4,7]$ (see also $[8,9]$ ). While the principle idea is straightforward, there are some more subtle issues in transferring the concept of excitable systems and stochastic resonance to small systems. For large clusters, action potentials are generated when the membrane potential exceeds a threshold value. As the ion channel cluster becomes smaller, the threshold softens and becomes conceptually irrelevant. The threshold-crossing criterion is replaced by a criterion based on channel kinetics [10]. Second, the relation between size and noise is not simple and monotonous. The spontaneous spiking rate as a function of the cluster size exhibits a maximum. A sequence of detailed studies of this novel form of stochastic resonance, for the particular case of clustered sodium and potassium channels, is being reviewed in this paper. In Sec. 1 we will briefly introduce the stochastic Hodgkin-Huxley model. In Sec. 2 we will discuss and evaluate some of the methods of solution. In Sec. 3 we review previous results and present some novel results.

\section{The Stochastic Hodgkin-Huxley Model}

We adopt the classic model for the ion channels introduced by Hodgkin and Huxley that mimics the potassium channel by four identical gates that stochastically switch between an open state and a closed state. The probabilities $p_{n}$ for the four gates $n=1,2,3,4$ to be open are described by the rate equations

$$
\dot{p}_{n}(t)=-\left(\alpha_{\mathrm{K}}(v)+\beta_{\mathrm{K}}(v)\right) p_{n}(t)+\alpha_{\mathrm{K}}(v),
$$

where $\alpha_{\mathrm{K}}(v)$ and $\beta_{\mathrm{K}}(v)$ are the membrane-voltage $v$ dependent opening and closing rates

$$
\alpha_{\mathrm{K}}(v)=\frac{0.01(10-v)}{\exp ((10-v) / 10)-1}, \quad \beta_{\mathrm{K}}(v)=0.125 \exp \left(-\frac{v}{80}\right) .
$$

The trans-membrane voltage $v$ is measured here and in all equations below in $\mathrm{mV}$ with respect to the physiologic cellular resting potential of $-65 \mathrm{mV}$. The potassium channel is open only when all four gates are open, i.e. with probability $p_{1} p_{2} p_{3} p_{4}$.

The sodium channel consists of four gates. Three identical fast gates increase their opening probability $q_{n}, n=1,2,3$ when the voltage $v$ becomes larger than the resting potential. The slower fourth deactivation gate decreases its open probability $q_{4}$ when the membrane potential increases. The gate variables obey the following rate equations:

$$
\begin{aligned}
& \dot{q}_{n}(t)=-\left(\alpha_{\mathrm{Na}}^{f}(v)+\beta_{\mathrm{Na}}^{f}(v)\right) q_{n}(t)+\alpha_{\mathrm{Na}}^{f}(v), \\
& \dot{q}_{4}(t)=-\left(\alpha_{\mathrm{Na}}^{s}(v)+\beta_{\mathrm{Na}}^{s}(v)\right) q_{4}(t)+\alpha_{\mathrm{Na}}^{s}(v),
\end{aligned}
$$


with the opening and closing rates

$$
\begin{aligned}
& \alpha_{\mathrm{Na}}^{f}(v)=\frac{0.1(25-v)}{\exp ((25-v) / 10)-1}, \quad \beta_{\mathrm{Na}}^{f}(v)=4.0 \exp \left(-\frac{v}{18}\right), \\
& \alpha_{\mathrm{Na}}^{s}(v)=0.07 \exp \left(-\frac{v}{20}\right), \quad \beta_{\mathrm{Na}}^{s}(v)=\frac{1}{\exp ((30-v) / 10)+1} .
\end{aligned}
$$

The membrane voltage is measured in $\mathrm{mV}$ with respect to the resting potential.

Although each individual ion channel opens and closes independently, the opening and closing rates (for the $n$ gate, they are $\alpha_{n}$ and $\beta_{n}$ ) are regulated by the same membrane potential. As a consequence, all ion channels are globally coupled through the membrane potential. For the density of the sodium and potassium channels (number of channels per area) we use $\rho_{\mathrm{Na}}=60 \mu \mathrm{m}^{-2}$ and $\rho_{\mathrm{K}}=18 \mu \mathrm{m}^{-2}$, respectively. The single-channel conductances of the sodium and potassium channels are given by $\gamma_{\mathrm{Na}}=\gamma_{\mathrm{K}}=20 \mathrm{pS}$. Using a membrane capacitance of $c=1 \mu \mathrm{F} / \mathrm{cm}^{2}[11]$ we end up with the following equation for the membrane potential

$$
\dot{v}=-\left(\frac{N_{\mathrm{K}}^{\mathrm{open}}}{\tau_{\mathrm{K}} N_{\mathrm{K}}}\left(v-v_{\mathrm{K}}^{\mathrm{rev}}\right)+\frac{N_{\mathrm{Na}}^{\mathrm{open}}}{\tau_{\mathrm{Na}} N_{\mathrm{Na}}}\left(v-v_{\mathrm{Na}}^{\mathrm{rev}}\right)+\frac{1}{\tau_{L}}\left(v-v_{l}\right)\right)+i_{\mathrm{ext}}(t),
$$

where $I_{\text {ext }}=c i_{\text {ext }}(t)$ denotes an externally applied current per area of the membrane and $v_{\mathrm{K}}^{\mathrm{rev}}=-12 \mathrm{mV}, v_{\mathrm{Na}}^{\mathrm{rev}}=115 \mathrm{mV}, v_{l}=10.6 \mathrm{mV}$ the reversal potentials of the potassium systems, sodium system and leakage system, respectively. The time constants are given by

$$
\begin{aligned}
\tau_{\mathrm{K}} & =\frac{1}{36} \mathrm{~ms}, \\
\tau_{\mathrm{Na}} & =\frac{1}{120} \mathrm{~ms}, \\
\tau_{L} & =3.3 \mathrm{~ms} .
\end{aligned}
$$

The numbers of open $\mathrm{K}^{+}$and $\mathrm{Na}^{+}$channels, $N_{\mathrm{K}}^{\text {open }}$ and $N_{\mathrm{Na}}^{\text {open }}$, respectively, have to be determined as a function of time by stochastic simulations with methods described in the next section.

The time scale of synaptic noise is about one order of magnitude smaller than channel noise (see e.g. in [12]). Thus we can consider synaptic noise as Gaussian white noise $\xi_{s}(t)$ with

$$
\begin{aligned}
\left\langle\xi_{s}(t)\right\rangle & =0, \\
\left\langle\xi_{s}(t) \xi_{s}\left(t^{\prime}\right)\right\rangle & =\sigma_{s}^{2} \delta\left(t-t^{\prime}\right),
\end{aligned}
$$

where $\sigma_{s}$ describes the strength of the synaptic noise. Since synaptic noise leads to elementary events in the synapse that are integrated, it has to be added to the right hand side of Eq. 5, i.e.

$$
\dot{v}=-\left(\frac{N_{\mathrm{K}}^{\mathrm{open}}}{\tau_{\mathrm{K}} N_{\mathrm{K}}}\left(v-v_{\mathrm{K}}^{\mathrm{rev}}\right)+\frac{N_{\mathrm{Na}}^{\mathrm{open}}}{\tau_{\mathrm{Na}} N_{\mathrm{Na}}}\left(v-v_{\mathrm{Na}}^{\mathrm{rev}}\right)+\frac{1}{\tau_{L}}\left(v-v_{l}\right)\right)+i_{\mathrm{ext}}(t)+\xi_{s}(t) .
$$




\subsection{Tracking the number of open ion channels}

In order to integrate Eq. 8, the number of open potassium and sodium channels needs to be determined. The simplest and most accurate method is to simulate each gate of each channel under the assumption that the opening and closing of the gates is a Markov-process. This method is inefficient since many simulated transitions of gates do not change the state of the channel (open or closed) and thus have no impact on the membrane voltage. More efficient schemes are based on representing the cluster of ion channels by the occupation numbers of channelstates. The potassium channel can be in five different states, $n_{0,1,2,3,4}$, which are characterized by $0,1,2,3,4$ gates open. Given the opening and closing rates of each gate by $\alpha_{\mathrm{K}}$ and $\beta_{\mathrm{K}}$ (see Eqs. 2), transition rates between the four states can be easily obtained. The kinetic scheme of a potassium channel is shown in Fig. 1. Similarly, the sodium channel, is characterized by the kinetic scheme in Fig. 2. The difference to the potassium channel is the slow deactivation gate that leads to a more complicated kinetic scheme.

$$
\left[n_{0}\right] \underset{\beta_{n}}{\stackrel{4 \alpha_{n}}{\rightleftarrows}}\left[n_{1}\right] \underset{2 \beta_{n}}{\stackrel{3 \alpha_{n}}{\rightleftarrows}}\left[n_{2}\right] \underset{3 \beta_{n}}{\stackrel{2 \alpha_{n}}{\rightleftarrows}}\left[n_{3}\right] \underset{4 \beta_{n}}{\stackrel{\alpha_{n}}{\rightleftarrows}}\left[n_{4}\right]
$$

Fig. 1. Kinetic scheme for a stochastic potassium channel.

$$
\begin{aligned}
& {\left[m_{0} h_{1}\right] \underset{\beta_{m}}{\stackrel{3 \alpha_{m}}{\rightleftarrows}}\left[m_{1} h_{1}\right] \underset{2 \beta_{m}}{\stackrel{2 \alpha_{m}}{\rightleftarrows}}\left[m_{2} h_{1}\right] \underset{3 \beta_{m}}{\stackrel{\alpha_{m}}{\rightleftarrows}}\left[m_{3} h_{1}\right]} \\
& \alpha_{h} \uparrow\left|\beta_{h} \quad \alpha_{h} \uparrow\right| \beta_{h} \quad \alpha_{h} \uparrow\left|\beta_{h} \quad \alpha_{h} \uparrow\right| \beta_{h} \\
& {\left[m_{0} h_{0}\right] \underset{\beta_{m}}{\stackrel{3 \alpha_{m}}{\rightleftarrows}}\left[m_{1} h_{0}\right] \underset{2 \beta_{m}}{\stackrel{2 \alpha_{m}}{\rightleftarrows}}\left[m_{2} h_{0}\right] \underset{3 \beta_{m}}{\stackrel{\alpha_{m}}{\rightleftarrows}}\left[m_{3} h_{0}\right]}
\end{aligned}
$$

Fig. 2. Kinetic scheme of a sodium channel.

The states of the sodium and potassium channels in the cluster are fully specified by the numbers of potassium channels $\left[n_{k}\right]$ in the states $n_{k}$ and the number of sodium channels $\left[m_{i} h_{j}\right]$ in the states $m_{i} h_{j}$. The number of activated potassium and sodium channels is given by $\left[n_{4}\right]$ and $\left[m_{3} h_{1}\right]$, respectively. Let, for example, the transition rate between the two states $S_{1}$ and $S_{2}$ be $\gamma_{S_{1} S_{2}}$ and the populations of these states be $\left[n_{1}\right]$ and $\left[n_{2}\right]$. Then, the probability $p$ that a channel switches within the time interval $(t, t+\delta t)$ from state $S_{1}$ to $S_{2}$ is given by $p=\gamma_{S_{1} S_{2}} \delta t$. The probability that, $\left[\delta n_{12}\right]=\left[n_{1}(t)\right]-\left[n_{1}(t+\delta t)\right]=\left[n_{2}(t+\delta t)\right]-\left[n_{2}(t)\right]$, channels switch from state $S_{1}$ to state $S_{2}$ in the same time interval satisfies the binomial distribution

$$
P\left(\left[\delta n_{12}\right]\right)=\left(\begin{array}{l}
{\left[n_{1}\right]} \\
{\left[\delta n_{12}\right]}
\end{array}\right) p^{\left[\delta n_{12}\right]}(1-p)^{\left(\left[n_{1}\right]-\left[\delta n_{12}\right]\right)} .
$$

Thus the number of switching channels between the states is sequentially drawn from binomial distributions. If the cluster of channels is large i.e. $\left[n_{1}\right]$ is large, the number of switching channels is also large in the average. Thus, in the time interval $\delta t$ larger channel clusters experience more transitions. 
In order to update the state of the population of ion channels with time, we have to create rules in what sequence the states are updated. The simple stochastic methods, described in the previous section, does not require such rules. In order to enforce positive occupation numbers we update the occupation numbers sequentially, starting with the process with the largest rate and so forth.

\subsection{Langevin approach}

Based on a the truncation of a Kramers-Moyal expansion of the master equations for single gates, Fox and $\mathrm{Lu}[13,14]$ have derived the following set of Ito-Langevin equations for the gating variables $n, m$ and $h$ for large ion channel clusters (i.e. when the number of channels in the cluster is large)

$$
\begin{aligned}
& \frac{d}{d t} n=\alpha_{n}(1-n)-\beta_{n} n+\bar{g}_{n}(t), \\
& \frac{d}{d t} h=\alpha_{h}(1-h)-\beta_{h} h+\bar{g}_{h}(t), \\
& \frac{d}{d t} m=\alpha_{m}(1-m)-\beta_{m} m+\bar{g}_{m}(t),
\end{aligned}
$$

where the variables $\bar{g}_{n}(t), \bar{g}_{h}(t), \bar{g}_{m}(t)$ denote Gaussian, zero-mean white noise with

$$
\begin{aligned}
\left\langle\bar{g}_{n}(t) \bar{g}_{n}\left(t^{\prime}\right)\right\rangle & =\frac{2}{N_{\mathrm{K}}} \frac{\alpha_{n} \beta_{n}}{\alpha_{n}+\beta_{n}} \delta\left(t-t^{\prime}\right), \\
\left\langle\bar{g}_{m}(t) \bar{g}_{m}\left(t^{\prime}\right)\right\rangle & =\frac{2}{N_{\mathrm{Na}}} \frac{\alpha_{m} \beta_{m}}{\alpha_{m}+\beta_{m}} \delta\left(t-t^{\prime}\right), \\
\left\langle\bar{g}_{h}(t) \bar{g}_{h}\left(t^{\prime}\right)\right\rangle & =\frac{2}{N_{\mathrm{Na}}} \frac{\alpha_{h} \beta_{h}}{\alpha_{h}+\beta_{h}} \delta\left(t-t^{\prime}\right) .
\end{aligned}
$$

Here $N_{\mathrm{K}}$ and $N_{\mathrm{Na}}$ denote the total number of potassium and sodium channels. It is necessary to include restriction to guarantee that $m, n$ and $h$ do not leave the unit interval $[0,1]$. The differential equation for the membrane potential is the classic Hodgkin-Huxley equations where $m^{3} h$ determines the fraction of open sodium channels and $n^{4}$ the fraction of open potassium channels, i.e.

$$
\dot{v}=-\left(\frac{1}{\tau_{\mathrm{K}}} n^{4}\left(v-v_{\mathrm{K}}^{\mathrm{rev}}\right)+\frac{1}{\tau_{\mathrm{Na}}} m^{3} h\left(v-v_{\mathrm{Na}}^{\mathrm{rev}}\right)+\frac{1}{\tau_{L}}\left(v-v_{l}\right)\right)+i_{\mathrm{ext}}(t)+\xi_{s}(t) .
$$

Equations (10), (11), (12) have to be integrated numerically in order to predict a neuronal spike train with the spikes occurrences at $t_{i}, i=0, \ldots, N$.

\section{Results}

\subsection{Spike rates}

While in the original, deterministic Hodgkin-Huxley model action potentials occur only for external current stimuli that exceed a threshold, the intrinsic channel noise can initiate spontaneous spikes even in the absence of external stimuli [15-19]. The 


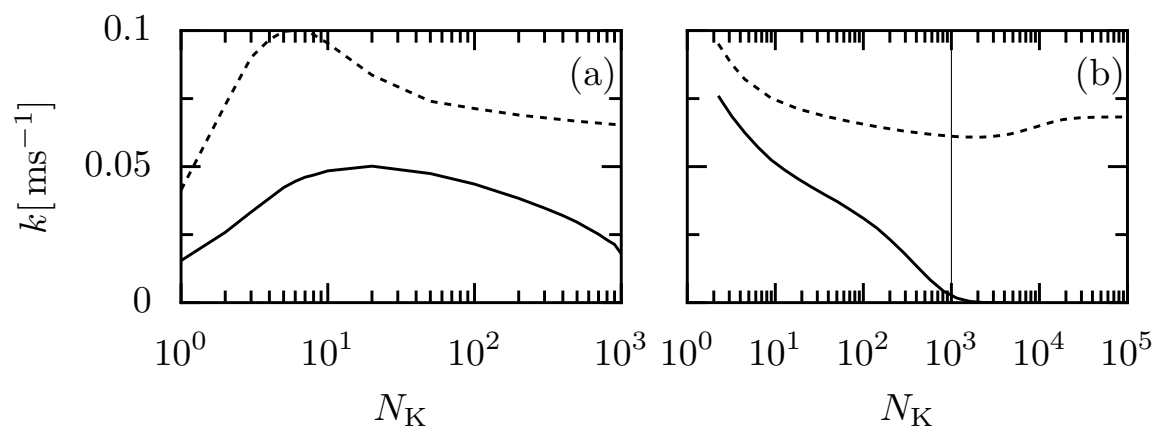

Fig. 3. The mean spiking rate $k$ which is given as the inverse of the mean interspike interval, cf. Eq. 13, is plotted against the number of potassium ion channels for $I_{\text {ext }}=0$ (solid lines) and $I_{\text {ext }}=10 \mu \mathrm{A} / \mathrm{cm}^{2}$ (dotted lines) using direct Markov simulations (a) and the Langevin approach (b). The vertical line in Fig. 3(b) corresponds to the maximal $N_{\mathrm{K}}$ in Fig. 3(a).

mean interspike interval, i.e.

$$
\langle T\rangle=\lim _{N \rightarrow \infty} \frac{1}{N} \sum_{i=1}^{N}\left(t_{i}-t_{i-1}\right),
$$

becomes a function of the number of ion channels. The inverse mean interspike interval defines the spiking rate $k=1 /\langle T\rangle$. Fig. 3 shows the dependence of the spiking rate $k$ on the number of potassium ion channels $N_{\mathrm{K}}$ (the number of sodium ion channels $N_{\mathrm{Na}}$ is kept proportional, $N_{\mathrm{Na}}=\frac{60}{18} N_{\mathrm{K}}$, to the number of potassium ion channels). For $I_{\text {ext }}=10 \mu \mathrm{A} / \mathrm{cm}^{2}$ the kinetic scheme simulations and the Langevin approach shows pretty good agreement except for small clusters $N_{\mathrm{K}} \lesssim 10$. For vanishing external current $\left(I_{\text {ext }}=0\right)$ the methods do not compare as well. Similar discrepancies between the master equation description and the corresponding Fokker-Planck approximation in a weak noise limit are known for a long time [20].

The spontaneous spiking rate $k$ becomes a non-monotonic function of the size of the cluster. It shows a distinct maximum near $N_{\mathrm{K}}=7$, cf. Fig. 3(a), within the stochastic kinetic scheme description. For a small number of ion channels, the stochastic opening of a single sodium channel results with large likelihood in an action potential. Therefore, the rate of spontaneous action potentials initially increases with the number of channels. For a larger number of ion channels, however, the common membrane voltage couples the random opening and closing events more tightly. This results in the collective events stemming from a larger portion of ion channels available at the same time. In the corresponding regime, an exponential decrease of the spiking rate occurs with the increasing patch size for a deterministically subthreshold driving $I_{\text {ext }}<6.26 \mu \mathrm{A} / \mathrm{cm}^{2}$. Since the generation of spontaneous action potentials is caused merely by the channel noise stemming from the fluctuations of the number of open ion channels, a decreasing noise level or an increasing number of ion channels, respectively, leads to a less probable activation of the cell membrane. With an additional, externally applied positive and constant current, the barrier crossing to excitation becomes generally more probable. This results in increasing spiking rate. In the regime of super-threshold constant 

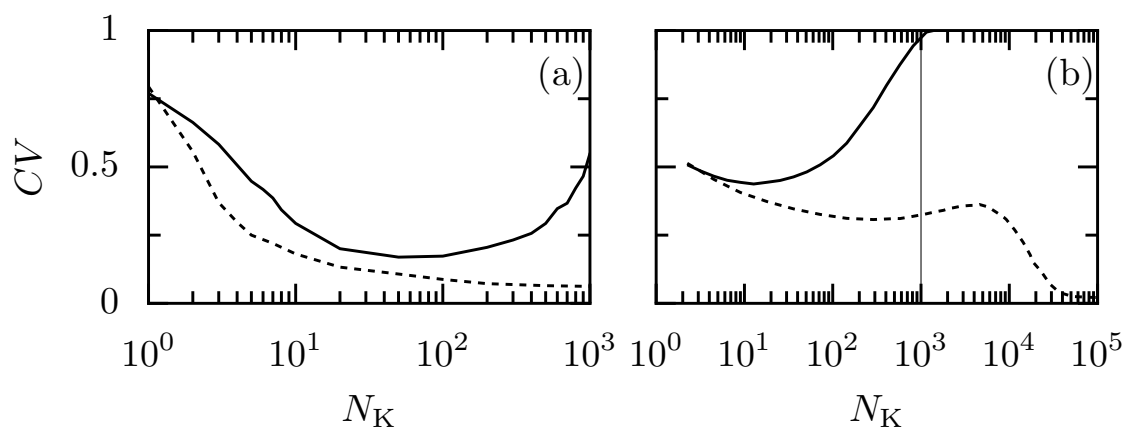

Fig. 4. Same figures as Fig. 3 but for the coefficient of variation $C V$, cf. Eq. 14 .

current driving $I_{\text {ext }} \geq 9.762 \mu \mathrm{A} / \mathrm{cm}^{2}$ a different effect takes place. Here, for large clusters, where the spiking events are determined by the excitable properties of the macroscopic Hodgkin-Huxley description of the neuron, the spiking rate decreases with increasing cluster sizes to approach an asymptotic value. For small cluster sizes it increases with increasing cluster size for firing events are determined by single-channel kinetics (see discussion above and Fig. 3(b)).

\subsection{Coherence resonance}

We next address the regularity of spontaneous action potentials. A proper measure is the coefficient of variation, $C V$, a measure of coherence, which is given as the ratio of the standard deviation of interspike time-intervals from the corresponding mean value to the mean value:

$$
C V=\frac{\sqrt{\left\langle T^{2}\right\rangle-\langle T\rangle^{2}}}{\langle T\rangle} .
$$

In (14), $\left\langle T^{2}\right\rangle:=\frac{1}{N} \sum\left(t_{i}-t_{i-1}\right)^{2}$ is the mean-squared interspike interval. For a fully disordered point process (the case of Poisson process) the coefficient of variation $C V$ assumes the value $C V=1$, while for more ordered processes it assumes smaller values and for a deterministic signal $C V$ vanishes.

Figure 4 depicts the coefficient of variation for different constant driving currents. The $C V$ reveals the phenomenon of intrinsic coherence resonance [3,4]. At an optimal dose of internal noise, i.e. an optimal number of ion channels, the $C V$ for $I_{\text {ext }}=0$ exhibits a minimum, where the spike train becomes distinctly more ordered. This maximum in temporal coherence occurs at a cluster size at which the spontaneous firing rate exhibits the maximum. For increasing applied external constant current the spike generation becomes more rhythmic. For a strong driving $I_{\text {ext }}>9.763 \mu \mathrm{A} / \mathrm{cm}^{2}, C V$ tends to zero in the limit of infinite numbers of ion channels, cf. Fig. 4(b).

\subsection{Stochastic resonance}

After studying the impact of constant current driving we focus next our attention on the effect of stochastic resonance (SR). We address the effect of genuine intrinsic 

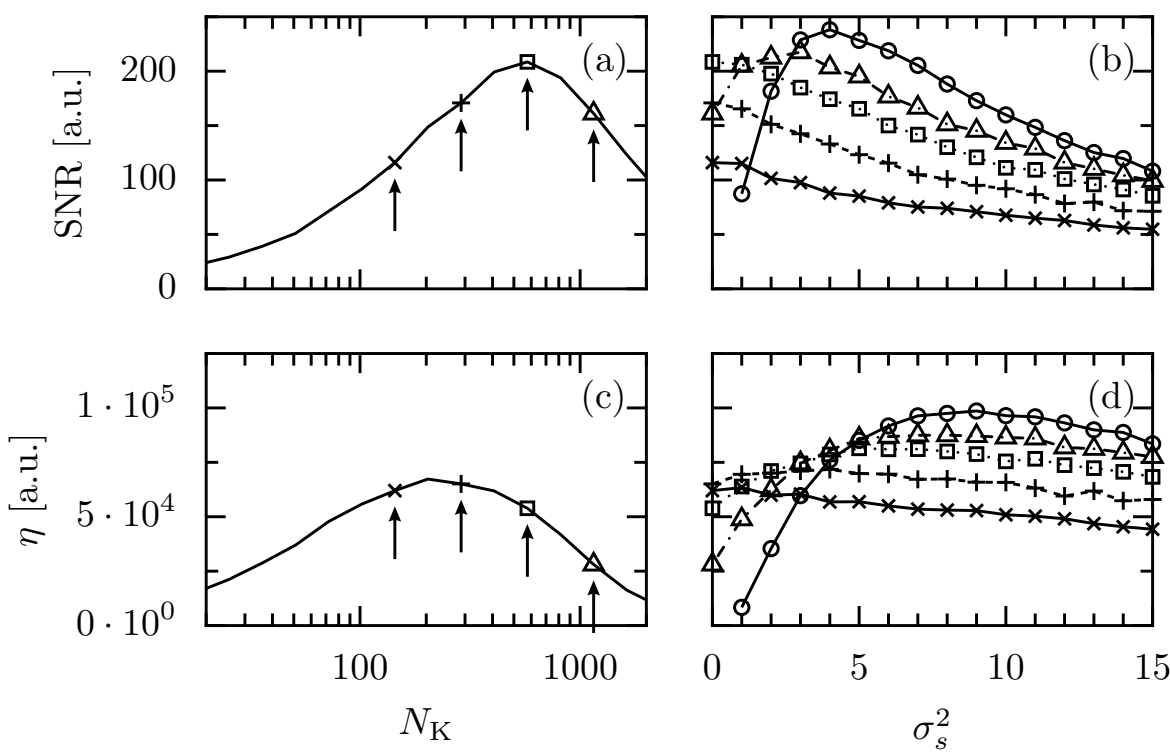

Fig. 5. The signal-to-noise ratio $S N R$ and the spectral amplification $\eta$ of an external subthreshold sinusoidal stimulus with amplitude $A=1.0 \mu \mathrm{A} / \mathrm{cm}^{2}$ and angular driving frequency $\Omega=0.3 \mathrm{~ms}^{-1}$ for different observation areas: (a) and (c) no synaptic noise; (b) and (d) the $S N R$ and $\eta$ versus the synaptic noise for the system sizes indicated by the arrows in (a) and (c): $N_{\mathrm{K}}=144$, solid lines through the crosses; $N_{\mathrm{K}}=288$, long dashed line connecting the pluses; $N_{\mathrm{K}}=576$, short dashed line through squares; $N_{\mathrm{K}}=1152$, dotted line connecting the triangles. The situation with no internal noise (i.e. formally $N_{\mathrm{K}} \rightarrow \infty$ ) is depicted by the dotted line connecting the circles.

stochastic resonance, where the response of the system to a subthreshold external stimulus is optimized solely due to internal, ubiquitous channel noise. Therefore, we apply a sinusoidal driving current to the cell membrane with the ion channel cluster:

$$
I_{\text {ext }}(t)=A \sin (\Omega t),
$$

with the driving amplitude $A$ and the angular driving frequency $\Omega$. Such a driving influences the spiking rate and the relative fluctuations of the spikes. A quantitative measure of the encoding of the sinusoidal signal in the spike train can be derived from the power spectrum $S(\omega)$ given as:

$$
S(\omega)=\lim _{T \rightarrow \infty} \frac{1}{T}\left|\sum_{n} \mathrm{e}^{-i \omega t_{n}}\right|^{2},
$$

with $T$ is the total integration time and the $t_{n}$ denote the times of spike occurrences. Most notably, we found a narrow peak at the driving frequency $\Omega$. Due to the finite length of the spike trains, this peak is not $\delta$-shaped, but is slightly broadened. From $S(\omega)$ we compute the signal-to-noise ratio $S N R$ and the spectral amplification $\eta$ (ratio of the powers at the frequency $\Omega$ of the spike train and the ac-signal).

In the absence of synaptic noise, cf. Fig. 5(a) and (c), we discover the effect of genuine intrinsic stochastic resonance, where the response of the system to the subthreshold external stimulus is optimized solely due to internal, ubiquitous noise. 
For the given parameters, $\mathrm{SR}$ in the spectral amplification of signal occurs for $N_{\mathrm{K}} \approx 180$ and in the signal-to-noise ratio for a different value $N_{\mathrm{K}} \approx 580$. Starting from $N_{\mathrm{K}} \approx 180$ the growing internal noise monotonically deteriorates the amplitude of system response at the driving frequency. Moreover, upon reaching $N_{\mathrm{K}} \approx 580$ it deteriorates the quality of signal transduction which is measured by $S N R$.

Under such circumstances, one would predict that the addition of an external noise (which corresponds to the conventional situation in biological SR studies) cannot improve $\eta$ and $S N R$ further, i.e. the conventional SR will not be exhibited. In order to verify this prediction, we add synaptic noise. The corresponding results, depicted in Fig. 5(b) and (d) fully confirm the above prediction. Conventional SR therefore occurs only for large ion channel clusters beyond an optimal size and it reaches a saturation in the limit $N_{\mathrm{K}} \rightarrow \infty$ (limit of the deterministic HodgkinHuxley model).

\section{Conclusions}

In conclusion, we have investigated stochastic resonance in a stochastic generalization of the Hodgkin-Huxley model. The spontaneous fluctuations of the ion channel conductance due to individual stochastic ion channel dynamics has been systematically taken into account by two methods: firstly, we have simulated the evolution of the membrane potential based upon a Markov-simulation of the kinetic of the potassium and sodium ion channels, secondly, we have followed a Langevin approach. Within the both methods we have shown that the excitable membrane patches exhibit a spontaneous spiking activity due to the omnipresent internal noise. The main results of this study refers to the phenomenon of intrinsic stochastic resonance. Here, the intrinsic channel noise alone give rise to SR (see Fig. 5). Moreover, SR induced by synaptic noise only occurs for sufficiently large clusters of ion channels, where the internal noise strength is not at its optimal value.

\section{Acknowledgement}

It is a pleasure to dedicate this work to Frank Moss on the occasion of his 70th birthday together with our best wishes for many more fruitful collaborations and scientific discussions. As a matter of fact, Frank is still young enough to appreciate and to contribute great science. G. Schmid, I. Goychuk and P. Hänggi gratefully acknowledge the support of this work by the Deutsche Forschungsgemeinschaft, SFB 486 Manipulation of matter on the nanoscale, Project A10. This research (P. Jung and S. Zeng) was also supported by the National Science Foundation under Grant No. IBN-0078055.

\section{References}

[1] C. Hildebrand and S. Waxman, Regional node-like membrane specializations in nonmyelinated axons of rat retinal nerve fiber layer, Brain Research 258 (1983) 23-32.

[2] C. Guo and H. Levine, A Thermodynamic Model for Receptor Clustering, Biophys. J. 77 (1999) 2358-2365.

[3] P. Jung and J. W. Shuai, Optimal size of ion channel clusters, Europhys. Lett. 56 (2001) 29-35. 
[4] G. Schmid, I. Goychuk and P. Hänggi, Stochastic resonance as a collective property of ion channel assemblies, Europhys. Lett. 56 (2001) 22-28.

[5] L. Gammaitoni, P. Hänggi, P. Jung and F. Marchesoni, Stochastic resonance, Rev. Mod. Phys. 70 (1998) 223-288; P. Hänggi, P. Talkner and M. Borkovec, Reaction-rate theory: fifty years after Kramers, Rev. Mod. Phys. 62 (1990) 251-342.

[6] K. Wiesenfeld and F. Moss, Stochastic resonance and the benefits of noise - from ice ages to crayfish and squids, Nature (London) 373 (1995) 33-36.

[7] G. Schmid, I. Goychuk and P. Hänggi, Channel noise and synchronization in excitable membranes, Physica A 325 (2003) 165-175.

[8] A. Pikovsky, A. Zaikin and M. A. Casa, System size resonance in coupled noisy systems and in the Ising model, Phys. Rev. Lett. 88 (2002), art. no. 050601.

[9] R. Toral, C. R. Mirasso and J. D. Gunton, System size coherence resonance in coupled FitzHugh-Nagumo models, Europhys. Lett. 61 (2003) 162-167.

[10] S. Zeng and P. Jung, Mechanism for neuronal spike-generation by small and large ion channel clusters, subm. to Phys. Rev. E.

[11] A. L. Hodgkin and A. F. Huxley, A quantitative description of the membrane current and its application to conductance and excitation, J. Physiol. (London) 117 (1952) $500-544$.

[12] A. Manwani and C. Koch, Detecting and estimating signals in noisy cable structures, Neural Computation 11 (1999) 1797-1873.

[13] R. F. Fox and Y. N. Lu, Emergent collective behavior in large number of globally coupled independently stochastic ion channels, Phys. Rev. E 49 (1994) 3421-3431.

[14] R. F. Fox, Stochastic versions of the Hodgkin-Huxley equations, Biophys. J. 72 (1997) 2068-2074.

[15] J. A. White, J. T. Rubinstein and A. R. Kay, Channel noise in neurons, Trends Neurosci. 23 (2000) 131-137.

[16] C. C. Chow and J. A. White, Spontaneous action potentials due to channel noise, Biophys. J. 71 (1996) 3013-3021.

[17] J. R. Clay and L. J. DeFelice, Relationship between membrane excitability and single channel open-closed kinetics, Biophys. J. 42 (1983) 151-157.

[18] A. F. Strassberg and L. J. DeFelice, Limitations of the Hodgkin-Huxley formalism: effects of single channel kinetics on transmembrane voltage dynamics, Neural Comput. 5 (1993) 843-855.

[19] L. J. DeFelice and A. Isaac, Chaotic states in a random world: relationship between the nonlinear differential equations of excitability and the stochastic properties of ion channels, J. Stat. Phys. 70 (1993) 339-354.

[20] P. Hänggi, H. Grabert, P. Talkner and H. Thomas, Bistable Systems: Master Equation Versus Fokker-Planck Modeling, Phys. Rev. A 29 (1984) 371-378; P. Hänggi and P. Jung, Bistability in Active Circuits: Application of a Novel Fokker-Planck Approach, IBM Journal of Research and Development 32 (1988) 119-125. 